\title{
Valoración de las herramientas de auditoría aplicables al peritaje contable
}

\author{
Assessment of the audit tools applicable to the accounting expertise \\ Avaliação das ferramentas de auditoria aplicáveis à perícia contábil
}

\author{
《 Mario Flórez Guzmán \\ mariohfg@hotmail.com \\ ORCID: 0000-0002-8468-4168 \\ Corporación Universitaria Remington, Colombia

\section{《ulia María Cortes Espitia} \\ Corporación Universitaria Remington, Colombia
}

\author{
《 David Alejandro Sánchez Mendoza \\ avid.sanchezm@campusucc.edu.co
}

Universidad Cooperativa de Colombia

\author{
《 Mercy Lilliana Borbón Hoyos \\ mnorbon@uniremington.edu.co \\ ORCID: 0000-0002-8349-635X
}

Universidad Cooperativa de Colombia

Recibido: 16-03-2018 / Revisado: 20-03-2018 / Aceptado: 30-03-2018 / Publicado: 10-09-2018

\section{RESUMEN}

El presente artículo tiene por objetivo valorar las herramientas de auditorías tradicionales o especializadas, aplicables al peritaje contable. En su labor de peritaje, el contador público utiliza herramientas de auditoría tradicionales que son las entrevistas, encuestas, cuestionarios, dictamen, trazas o huellas, observación, muestreo, cuaderno de campo y las herramientas especializadas (software); Zifra, ACD Auditor, ACL, IDEA. Metodológicamente es un estudio de tipo documental, donde a través de fuentes secundarias de información se obtiene información sobre las herramientas que el auditor usa en su encargo, -tradicionales y especializadas-Se concluye que las herramientas son de vital importancia en una auditoria, dan aval y fiabilidad a los estados financieros de cada empresa, en base a la actualización constante de nuestro mundo.

Palabras clave: Herramientas de auditoría; peritaje contable, software de auditoria

ABSTRACT

RESUMO

The objective of this article is to assess traditional or specialized auditing is tools, applicable to accounting expertise. In his expert work, the public accountant uses traditional auditing tools which are interviews, surveys, questionnaires, opinion, traces or traces, observation, sampling, field notebook and specialized tools (software); Zifra, ACD Auditor, ACL, IDEA. Methodologically, it is a documentary-type study, where, through secondary sources of information, information is obtained on the tools that the auditor uses in his assignment, - traditional and specialized-. It is concluded that the tools are of vital importance in an audit, they give endorsement and reliability to the financial statements of each company, based on the constant updating of our world.

Key words: Audit tools; accounting expertise, audit software
O objetivo deste artigo é avaliar as ferramentas tradicionais ou especializadas de auditoria, aplicáveis à perícia contábil. Em seu trabalho pericial, o contador público utiliza ferramentas tradicionais de auditoria que são entrevistas, pesquisas, questionários, opinião, traços ou traços, observação, amostragem, caderno de campo e ferramentas especializadas (software); Zifra, Auditor ACD, ACL, IDEA. Metodologicamente, trata-se de um estudo do tipo documental, onde, através de fontes secundárias de informação, se obtém informação sobre as ferramentas que o auditor utiliza na sua missão, tradicionais e especializadas-. Conclui-se que as ferramentas são de vital importância em uma auditoria, pois dão respaldo e confiabilidade às demonstrações financeiras de cada empresa, com base na atualização constante do nosso mundo.

Palavras-chave: Ferramentas de auditoria; perícia em contabilidade, software de auditoria 


\section{INTRODUCCIÓN}

$\mathrm{L}$ a profesión contable se desarrolla por sus fronteras multifacéticas, y el pico que históricamente ha marcado su avance es la auditoría. La evolución de la auditoría es resultado de la responsabilidad social que se le ha otorgado, donde se materializa en la fe pública.

Cuando el contador público brinda fe pública emite una garantía personal basada en sus cualidades profesionales, la auditoría informa del estado real de un asunto de interés, gracias a la confianza que brinda el contador público cuando es representante de la fe pública.

Para ello, el contador, tiene a su disposición herramientas de auditoría tradicionales y especializadas, de vital importancia en encargo financiero, encargo que, sin lugar a dudas da aval y fiabilidad a los estados financieros de cada empresa, en base a la actualización constante de nuestro mundo se fueron creando ciertas herramientas especializadas o software para ayudar al auditor a incrementar su productividad y su efectividad, además ayuda a que las auditorias se efectúen con un mayor valor agregado.

Es así, que la auditoría aplica en un peritaje contable surge en el momento en que las auditorias tradicionales no son suficientes y nace esta con los intentos por detectar e investigar el fraude en los estados financieros y posteriormente ha ido ampliando su campo de acción en la medida que ha desarrollado técnicas específicas para combatir la ilegalidad y trabaja estrictamente en la atención de la justicia.

El encargo de auditoría es particularmente dependiente de los computadores para desarrollar muchas de sus funciones, todos los tipos de auditorías y de auditores pueden tomar ventaja de las herramientas de software y de las técnicas para ser más eficientes y eficaces.

En este orden de ideas, las herramientas de auditoría sean tradicionales o especializadas juegan un papel muy importante para la identificación de los riesgos, la adecuada investigación y pronta detección en los errores o en el mayor de los casos la detección de fraudes en cada una de las auditorias ejercidas.

\section{Marco teórico}

\section{Importancia de las herramientas tradicionales y especializadas en un encargo de auditoría}

Con las herramientas tradicionales de auditoría, se genera un aval y fiabilidad a los estados financieros y supone un gran apoyo para la gestión diaria de la empresa, sin desconocer que las auditorias se pueden realizar de manera distinta dependiendo del profesional; algunos prefieren utilizar las herramientas de auditoría tradicionales y otros prefieren las herramientas especializadas.

Estas herramientas tradicionales son las que cada profesional va forjando y emplea a través de su experticia profesional, sin la ayuda de un software para su uso; algunas de ellas son, cuestionarios entrevista o encuesta, dictamen, trazas o huellas, observación, muestreo, cuaderno de campo y 
cumplen una importante labor, ya que otorgan al auditor pruebas sobre el funcionamiento del área o proceso auditado.

La entrevista o encuesta es aquella herramienta que facilita al auditor un análisis de la situación de la empresa que va a ser auditada, además puede ser una herramienta de gran ayuda debido a que con esto pueden conocer la verdad o si el funcionario está ocultando información valiosa para la auditoria, como lo expresa Tello (2012).

La entrevista, junto con la encuesta, es otra de las técnicas utilizadas en las auditorías en comunicaciones. La entrevista no sólo sirve como complemento de la encuesta, correctamente utilizada posibilita un entendimiento profundo de las fortalezas y debilidades comunicativas de las organizaciones, al permitir que el auditor profundice en los temas que considere relevantes para su investigación (p.2).

El profesional del encargo de auditoría utiliza los cuestionarios, ya que por medio de este permite apoyar la gestión del auditor y le proporcionan un base para determinar un diagnóstico que, para la empresa, según la NIA 300 el auditor debe realizar una planeación de auditoría, por lo tanto, el objetivo principal del cuestionario es documentar la planeación de auditoría: en este se debe incluir el alcance, los objetivos, los recursos asignados para el proceso y los tiempos de ejecución de labores.

La auditoría se apoya en una de las herramientas más antiguas que existen y que en determinados casos llegar a ser pieza clave en un dictamen, cuando un auditor llegar a una empresa a realizar su trabajo el objetivo principal es realizar una evaluación del entorno para poder obtener información que le permita entender el fin de la empresa.

Los cuestionarios juegan un papel muy importante en una auditoria, ya que sirven para conocer qué grado de control maneja la entidad y la participación de los colaboradores de la organización con el mantenimiento de un ambiente de control, teniendo en cuenta que trabaja de la mano de otras técnicas (Actualicese, 2017). Esta herramienta le permite al auditor dar veracidad en que la información que obtuvo fue dada por una persona capacitada, autorizada y conocedora de los movimientos de la empresa.

Las trazas o huellas se utilizan para comprobar la ejecución de las validaciones de datos previstos por el auditor. Estas trazas o huellas no deben de afectar en lo absoluto el sistema de trabajo de la empresa, en dado caso que afecte se debe de acordar un horario en bien de las dos partes.

El auditor debe de estar atento a cualquier actitud o cambio repentino en la empresa, dado que pueden estar ocultando información valiosa para el proceso esto se puede determinar gracias a una herramienta tan básica como lo es la observación. Esta herramienta tradicional se usa en la planeación, ejecución, informe y monitoreo; es decir en todo el encargo.

El auditor no está obligado a examinar todas las áreas de la empresa o todas las transacciones que haga la misma, esto gracias a que él puede tomar una muestra representativa de las áreas o de las transacciones para poder obtener la evidencia que se requiera para su dictamen; teniendo en cuenta la 
materialidad de las transacciones y la importancia relativa que le dé a su juicio. El muestreo se define también como la aplicación de procedimientos de auditoría a menos del $100 \%$ de las transacciones o cuentas seleccionadas, de tal manera que todas las unidades de estudio, presenten la misma probabilidad de ser seleccionadas (Frett, 2016)

Un elemento importante como lo es el cuaderno de campo, el cual sirve para la toma de nota de cada uno de los hallazgos obtenidos en todas las herramientas anteriores, con el fin de apoyar en la toma de una decisión durante todo el proceso de la auditoria.

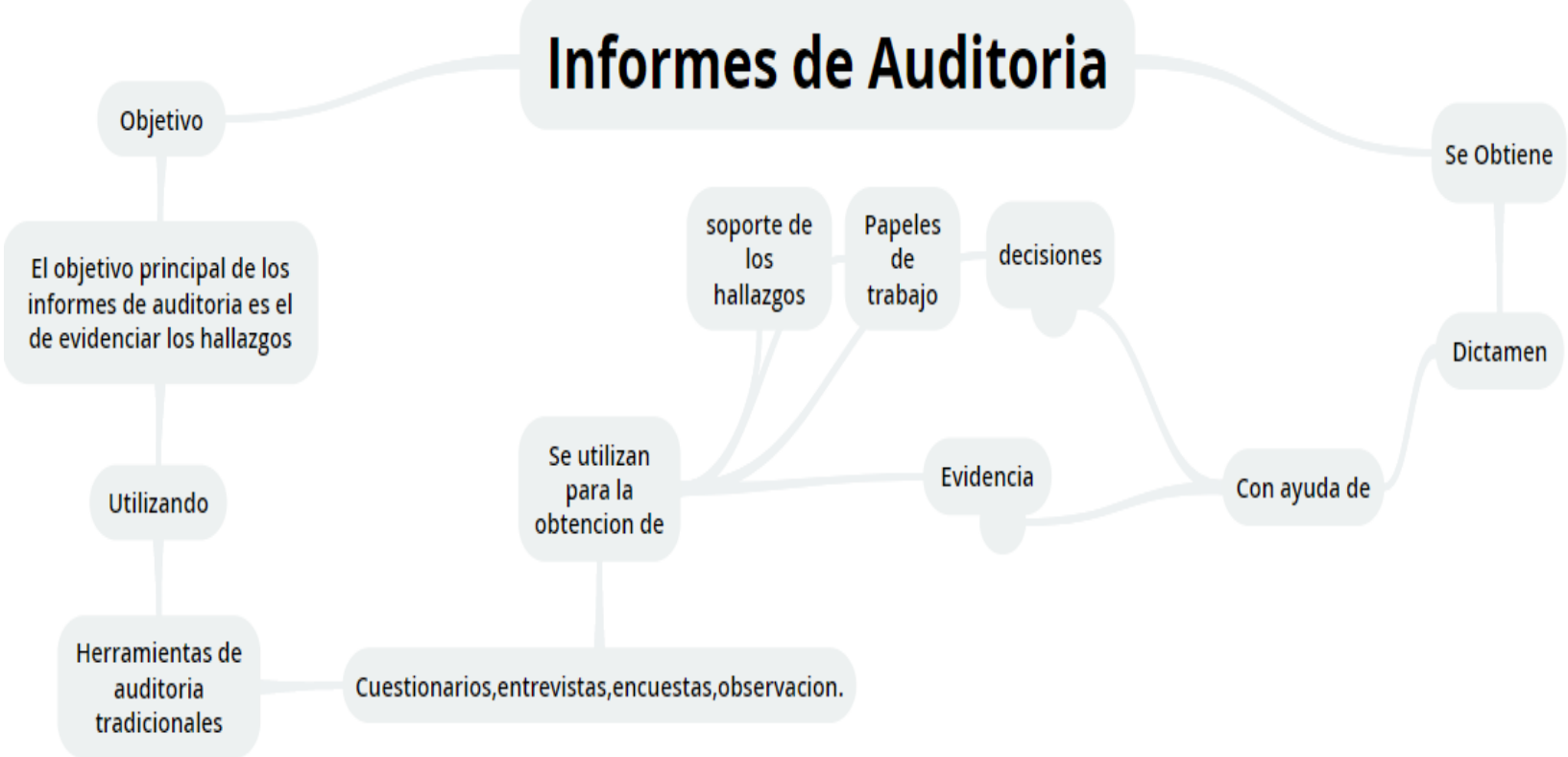

Figura 1. Mapa mental de herramientas tradicionales utilizadas en un Informe de auditoria

La contabilidad de papel ya se encuentra en un segundo plano, solo se ve en muy raras ocasiones, en una microempresa que maneje muy pocas ventas. Ahora bien, las empresas cada día están automatizando los procesos, gracias a los Software integrados que acumulan información y se compila en el sistema de información de la organización.

De esta forma, las herramientas especializadas, usadas por el auditor en su encargo se convierten en la mejor estrategia de tomar esa información sistematiza y a través de cálculos, mostrar las debilidades, errores u omisiones en la información. Estas son definidas como aquellas que ayudan al auditor a incrementar la productividad y efectividad del auditor, -por el uso de software-otorgando un mayor valor agregado. Estas otorgan mayor fiabilidad para elevar la calidad de la auditoria, puesto que no solo se basa en la experiencia del auditor, sino que, a través de las mismas, se generan resultados importantes, que pueden conducir la auditoría. 
Al respecto Duque y Tamayo (2012), manifiestan que:

La profesión de auditoría es particularmente dependiente de los computadores para desarrollar muchas de sus funciones, todos los tipos de auditorías y de auditores pueden tomar ventaja de las herramientas de software y de las técnicas para ser más eficientes y eficaces (p.101).

Hoy en día, existen diversas herramientas que pueden ayudar o facilitar el trabajo del auditor, debido a que se puede obtener ayuda de las herramientas especializadas, con Software especializado para una mayor precisión y procesamiento de la información que pueden ayudar en el dictamen final de la auditoria.

En este orden de ideas, existen diversos software o herramientas especializadas pero las más conocidas son como; Zifra, ACD Auditor, ACL, IDEA.

Zifra, que es desarrollado en España, denominado el más práctico del momento y fue desarrollado por y para auditores, cumple con los más altos requisitos de seguridad y confidencialidad, configurado para no permitir accesos indeseados a los documentos; con una base de datos cerrada al exterior, control de acceso al sistema por usuario y contraseña y limitación de acceso a cada auditoría sólo al equipo de trabajo, muestreos, planificación automática, programas de trabajo por área.

De igual manera, el $A C D$ auditor, es un software totalmente adaptado a las necesidades actuales de calidad que exigen las directivas de la Unión Europea y es reconocido por los auditores de todo el mundo como uno de los más potentes del mercado además permite la elaboración de los papeles de trabajo completando su contenido de forma automática en base a los procedimientos de auditoría típico o personalizado; este permite un muestreo estadístico.

El Software $A C L$, es el único software que integra a la perfección las capacidades de análisis de datos estándar de la industria en una evaluación integral de riesgos, gestión de auditorías, documentos de trabajo, problemas de flujo de trabajo de seguimiento y remediación.

Uno de los estudios respecto a estas herramientas especializadas de auditoría lo han realizado Martínez, Alfonso, y Marichal, (2012) argumentando que:

Esta herramienta reduce el riesgo y asegura el retorno de la inversión, también posee una poderosa combinación de accesos a datos, análisis y reportes integrados, $\mathrm{ACL}$ lee y compara los datos permitiendo a la fuente de datos permanecer intacta para una completa integridad y calidad de los mismos (p.12)

Entre las características más importantes que tiene el Software ACL se encuentran los análisis de datos, menor costo de adquisición, incremento en la calidad y la productividad e informes más detallados y confiables para una toma de decisiones con mayor seguridad.

IDEA, es una herramienta de software para el análisis de datos y permite visualizar la relación entre diferentes bases de datos, para lograr la efectividad en el análisis de las cifras; También permite hacer 
cruces de información, revisar facturas duplicadas, segmentar la información, realiza tareas de cálculo y análisis, de tal forma que sea muy fácil de revisión.

Otra definición del Software IDEA es como lo describe case ware analytics "es una herramienta de análisis completa, potente y fácil de usar que analiza rápidamente el 100\% de sus datos, garantizando su integridad, acelera su trabajo y prepara el terreno para auditorías más rápidas y efectivas".

Los tableros de Control, nacen con el objetivo de relacionar las principales figuras de la empresa dando a conocer al mundo la unificación de estrategias o planes de certificación mundial (ISO9000), lo explica (Flórez, Gallego y Hernández, 2015)"las anteriores herramientas focalizan sus funciones en la ayuda a la auditoría financiera de esta manera cobra valiosa importancia las herramientas especializadas de auditoría todo por que facilitan el trabajo que realiza el auditor aunque también ayuda para establecer un control o monitoreo continuo sobre la labor (p.665).

\section{Uso de las herramientas de auditoría en un peritaje contable}

Las herramientas de auditoría fueron diseñadas para analizar y optimizar los tiempos y esfuerzos de revisión, disminuir el volumen de trabajo y garantizar la minimización del riesgo, además que ayuda a mantener y defender los intereses de la compañía. Las herramientas de auditoría también fueron diseñadas para poder identificar los factores de riesgo en ciertas áreas de la empresa y logar como Objetivo principal el control de todos los riesgos.

Una herramienta de auditoría es el conjunto de elementos que permiten llevar a cabo las acciones definidas en las técnicas; estas son los métodos prácticos de investigación y prueba que el auditor utiliza para lograr la información y comprobación necesaria para poder emitir su opinión profesional (Alatrista, 2015).

El contador público para poder ejercer como auditor en un peritaje contable, debe acreditar su competencia profesional (Título universitario) es quien la ley faculta para dar Fe Pública y poder dictaminar la información económica y financiera de una empresa.

La Ley 43 de 1990, define a un contador público como "la persona natural que, mediante la inscripción que acredite su competencia profesional en los términos de la presente, está facultada para dar fe pública de hechos propios del ámbito de su profesión, dictaminar sobre estados financieros, realizar las demás actividades relacionadas con la ciencia contable en general (Ley 43 de 1990, art.1).

El contador público al momento de ejercer la auditoria en un peritaje contable, debe planificar sus actividades, para que se abarquen todas las consideraciones necesarias al momento de ejecutar el encargo, cuyo fin es recoger todas las evidencias - pruebas-, las cuales sirven de base para emitir su dictamen, que servirá de base ante un proceso judicial. 


\section{MÉTODO}

$\mathrm{L}$ a investigación fue un estudio descriptivo, de corte documental, basado en fuentes secundarias de información donde se analizaron las herramientas de auditorías especializadas y tradicionales, usadas por el Contador Público en su labor de perito auxiliar de la justicia. En esta investigación se tuvo en cuenta las siguientes fases: Fase 1. Planeación, Fase 2 desarrollo del programa de auditoría forense, Fase 3 comunicación de resultados, Fase 4 seguimiento monitoreo.

\section{Valoración de las herramientas de auditoria en un peritaje contable}

Las herramientas de auditoría se aplican en el peritaje contable siguiendo ciertas fases, planeación, desarrollo del programa de auditorita, comunicación de resultados, seguimiento y monitoreo.

En la primera fase, está la planeación y como lo estipulan las normas internacionales de auditoría emitida por la Federación Internacional de Contadores (IFAC); el auditor tuvo que planear la auditoria mediante un memorando de planeación, según lo explica la Contraloría de Bogotá el memorando de planeación, "es el documento a través del cual se realiza toda la programación de la auditoria. Constituye la carta de navegación del equipo auditor para llevar a cabo la auditoria gubernamental con enfoque integral" (p.2)

El auditor debe planear la auditoría con una actitud de sospecha profesional reconociendo que pueden existir circunstancias que causen que los estados financieros estén principalmente representados en forma errónea.

Para la primera fase, también se utilizó el Contrato de aseguramiento, que según lo indica Cuellar (2017), está enfocado en proporcionar un marco conceptual global dirigido a los trabajos de aseguramiento que tienen la intención de proveer un nivel de seguridad ya sea alto o moderado.

Para esto, el auditor tuvo que basar en la NIA 335 y conocer su alcance el cual estuvo establecido en identificar y valorar los riesgos en de incorrección en los estados financieros.

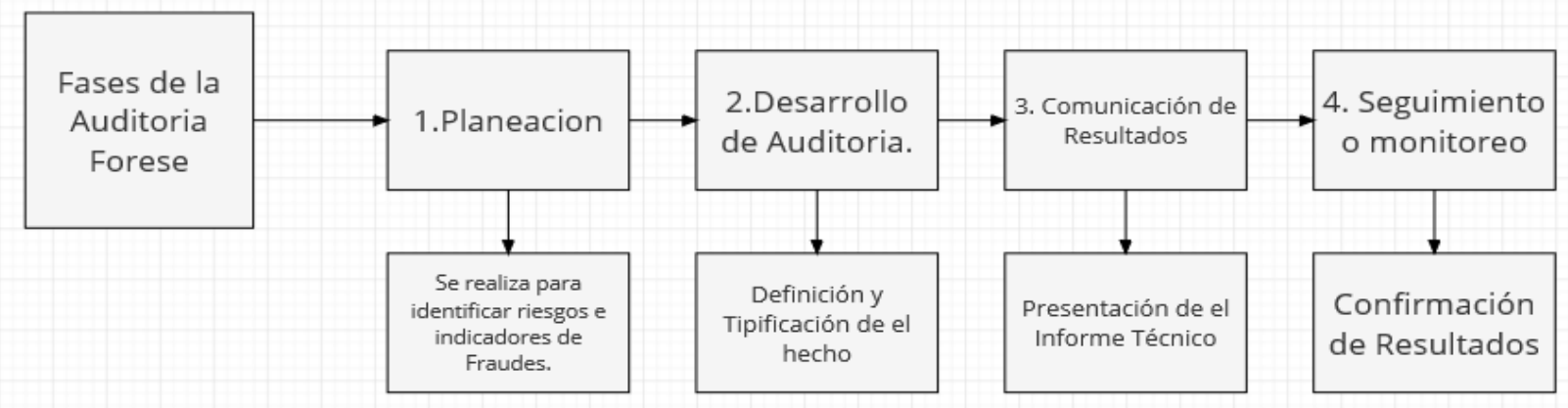

Figura 2. Fases de la auditoría 
Para la segunda fase, se tuvo que planificar el plan de trabajo, la elaboración y desarrollo del programa de auditoría forense y esta fase consta de 5 etapas de auditoría las cuales estuvieron distribuidas como la tipificación del hecho, la recopilación de evidencias, la cadena de custodia de las evidencias de auditoría, evaluación de evidencias y para finalizar el informe de la auditoria forense.

Para el informe final que da el auditor debe de estar muy bien preparado como lo explica el (Ministerio de Comercio, 2017) en el Decreto 2170 "el auditor concluirá si ha obtenido una seguridad razonable sobre si los estados financieros en su conjunto están libres de incorrección material, debida a fraude o error" (p.6).

La tipificación del hecho o la identificación del tipo de fraude según lo explica la NIA 240, 2013 "un acto intencionado realizado por una o más personas de la dirección, los responsables del gobierno de la entidad, los empleados o terceros, que conlleve la utilización del engaño con el fin de conseguir una ventaja injusta o ilegal" (p.5).

En este orden de ideas, la NIA 240 se convierte en una herramienta que permite identificar los factores de riesgo de fraude como aquellos "hechos o circunstancias que indiquen la existencia de un incentivo o elemento de presión para cometer fraude o que proporcionen una oportunidad para cometerlo" (IFAC, 2009).

La recopilación de evidencias consiste en obtener material probatorio para ser analizados según la ley, en alguno de los casos se hace necesaria la intervención de profesionales del derecho como expertos que soporten los procedimientos por lo general administrativos y que interpreten jurídicamente las leyes locales e internacionales

Analizando la variación de las herramientas de auditoría aplicables a un peritaje contable dice la NIA 230 la responsabilidad que tiene el auditor en preparar toda la información necesaria correspondiente a la auditoria y para esto tiene que utilizar todas las herramientas tradicionales de auditoría.

A partir del 22 de diciembre de 2017 las responsabilidades que tienen todos los contadores públicos que ejerzan como auditores según el (Decreto 2170, 2017) dice que tiene como objetivo "obtener una seguridad razonable de que los estados finj:inr,ieros en su conjunto están libres de incorrección material, debida a frAude (1 error, y emitir un informe de auditoría que contiene nuestra opinión" (P.24)

Como lo dicen las NIA la evidencia se convierte en un elemento obligatorio para el proceso de la investigación y como se expresa en la NIA 520, 2013 la evidencia "la evidencia de auditoría incluye tanto la información contenida en los registros contables de los que se obtienen los estados financieros, como otra información" (p.2). 
Tabla 1- Herramientas de auditoria en un peritaje contable

\begin{tabular}{|c|c|c|c|c|c|c|}
\hline \multirow[b]{2}{*}{$\begin{array}{l}\text { Tipo de } \\
\text { Riesgo }\end{array}$} & \multicolumn{5}{|c|}{$\begin{array}{c}\text { MATRIZ DE RIESGO } \\
\text { HERRAMEINTAS DE AUDITORÍA EN UN PERITAJE CONTABLE }\end{array}$} & \multirow[b]{2}{*}{$\begin{array}{c}\text { Clase de } \\
\text { Riesgo }\end{array}$} \\
\hline & Valoración & Nivel & Norma & Tipo de Riesgo & Porcentaje & \\
\hline $\begin{array}{c}\text { Riesgo } \\
\text { Humano }\end{array}$ & Alta & 3 & NIA 520 & $\begin{array}{l}\text { Se porduce cuando el personal de la } \\
\text { empresa comete errores en el manejo de } \\
\text { la información contable o por el no } \\
\text { correcto uso de las herrameintas. }\end{array}$ & $100 \%$ & $\begin{array}{c}\text { Riesgo } \\
\text { Inherente }\end{array}$ \\
\hline $\begin{array}{l}\text { Riesgo en } \\
\text { Auditoría }\end{array}$ & Bajo & 1 & $\begin{array}{l}\text { NIA } 200 \\
\text { Párrafo } \\
23\end{array}$ & $\begin{array}{l}\text { Se produce cuando el auditor expresa una } \\
\text { opinión de auditoría inapropiada cuando } \\
\text { los estados financieros estan erroneos. }\end{array}$ & $20 \%$ & $\begin{array}{r}\text { Riesgo de } \\
\text { Control }\end{array}$ \\
\hline $\begin{array}{l}\text { Riesgo en } \\
\text { Auditoría }\end{array}$ & Baja & 1 & NIA 500 & $\begin{array}{l}\text { Se produce cuando el cliente ejerce una } \\
\text { presión inadecuada para poder acomodar } \\
\text { el dictamen de el auditor a su favor. }\end{array}$ & $10 \%$ & $\begin{array}{c}\text { Riesgo } \\
\text { Inherente }\end{array}$ \\
\hline Siniestro & Baja & 1 & NIA 240 & $\begin{array}{l}\text { Puede ocurrir cuando se llegase a } \\
\text { presentar fuego, inundación o alguna clase } \\
\text { de desastre natural y con esto se perdería } \\
\text { toda la información }\end{array}$ & $10 \%$ & $\begin{array}{c}\text { Riesgo } \\
\text { Inherente }\end{array}$ \\
\hline
\end{tabular}

En la siguiente imagen se representa la importancia y la relación de las NIA referente a las evidencias de Auditoria. 


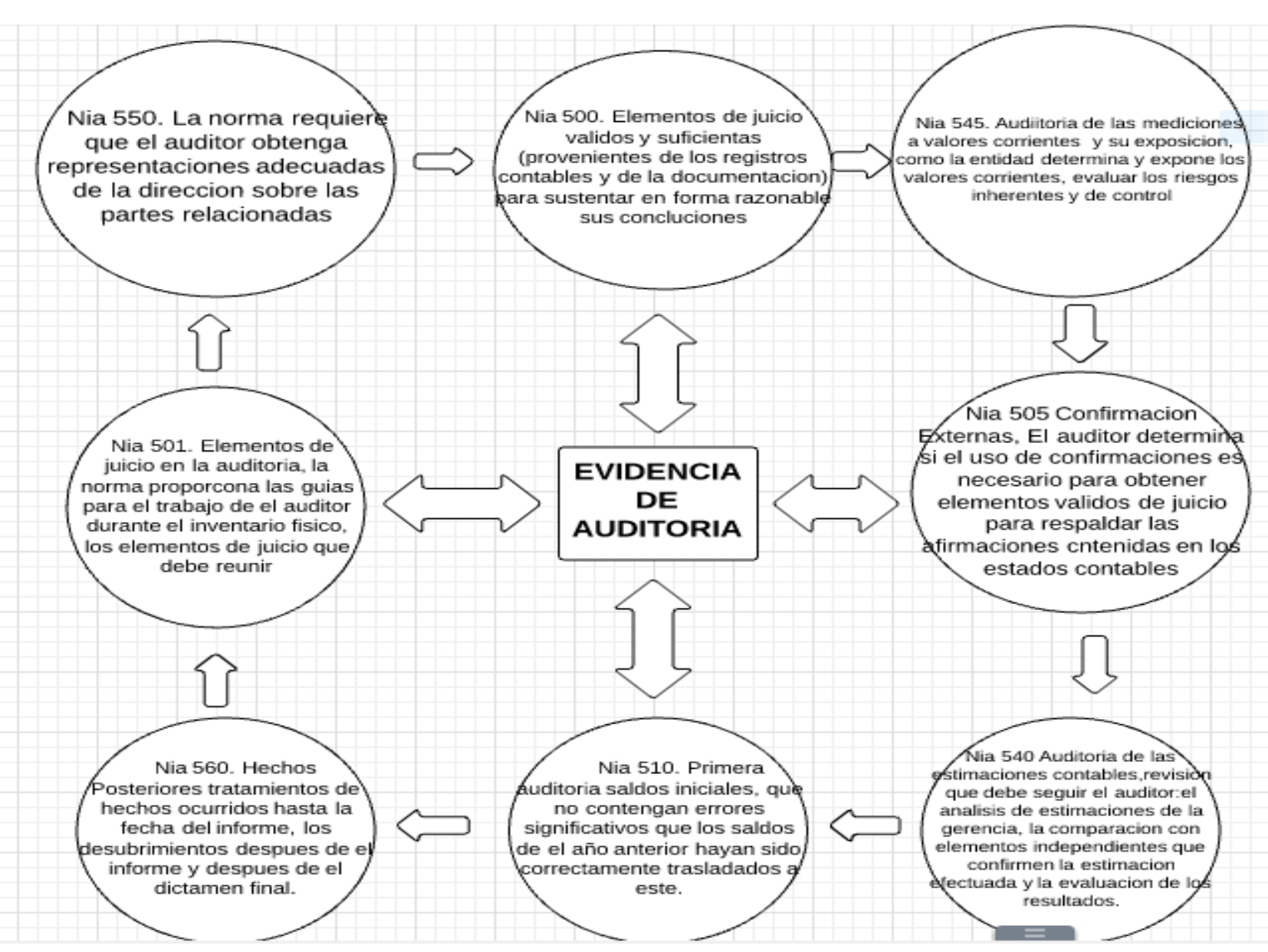

Figura 3. NIA en torno de la evidencia de auditoría.

En la tercera etapa, la cadena de custodia de la información obtenida en el peritaje contable inició en el lugar donde se descubrieron los materiales probatorios, seguido por la custodia el embalaje y envió -si es el caso- y tener por escrito los cambios que cada custodio realice a estos, este proceso finaliza por una orden de la autoridad competente.

Esta documentación para la auditoria es muy útil debido a que puede facilitar la planificación y realizar las revisiones en cuanto a los requerimientos legales; el objetivo principal de esta norma es preparar toda la documentación necesaria mediante el uso de las herramientas tradicionales de auditoría para poder obtener suficiente base para el informe final.

Para finalizar, con la tercera etapa, señala que para demostrar la autenticidad de los elementos materiales probatorios y la evidencia física se dará uso a la cadena de custodia; este proceso que garantiza la veracidad de la información recolectada, se inicia en el lugar o sitio donde fueron hallados los elementos de prueba seguido del embalaje-envío, y finalizando por orden de la autoridad competente quien se encargará de certificar la cadena de custodia. 
Ya como lo menciona la NIA 520 (IFAC, 2009) para la cuarta etapa esta norma suministró guías para la aplicación de procedimientos de estudio analítica en las etapas de planeación, recopilación de elementos de juicio es decir como pruebas reales y a la ejecución de la auditoría como una revisión global.

La decisión del auditor sobre qué procedimientos debería utilizar dependerá, según su juicio profesional, de su expectativa de efectividad y eficiencia de los procedimientos disponibles. Es decir, no hay una "fórmula secreta" sobre qué procedimientos utilizar y de qué tipo. Esto dependerá de qué tan efectivo y eficiente sea el procedimiento (que proporcione evidencia de auditoría y fácil de aplicar); es decir, que se logre cumplir con los objetivos de la auditoría, de la manera más rápida y eficiente.

La quinta y última etapa, fue la de seguimiento y monitoreo en donde es de conocimiento de todos que el dictamen debe ser claro, preciso y detallado; en él se darán a conocer los análisis, experimentos e investigaciones efectuados, pero cual es propósito de la NIA 700 quien es la que establece guías y dicta los lineamientos sobre el cómo realizar y que debe de contener el dictamen del auditor, emitido como resultado de una auditoría practicada por un auditor independiente de los estados financieros de una entidad (IFAC, 2009).

El informe o dictamen pericial reconoce ante la justicia demostrar por medios escritos $\mathrm{y} / \mathrm{o}$ documentales una conclusión sobre la verdad de un ilícito que lleva a esta a declarar la culpabilidad o inocencia del acusado. Los peritos realizarán el estudio acucioso, riguroso del problema encomendado para producir una explicación consistente; esa actividad cognoscitiva será condensada en un documento que refleje las secuencias fundamentales del estudio efectuado, los métodos y medios importantes empleados, una exposición razonada y coherente, las conclusiones, fecha y firma.

Según Congreso de la República, 2012 todo dictamen pericial debe de contener: a) La descripción de la persona, objeto o cosa materia de examen o estudio, así como, el estado y forma en que se encontraba. b) La relación detallada de todas las operaciones practicadas en la pericia y su resultado. c) Los medios científicos o técnicos de que se han valido para emitir su dictamen y d) Las conclusiones a las que llegan el (los) perito (s).

\section{RESULTADOS}

\section{Contraste de las herramientas de auditorías tradicionales y especializadas en un peritaje contable}

Hoy en día la auditoria ha especializado su aplicación, ya que ha surgido la necesidad de dar respuesta a las problemáticas del sector empresarial (industria, comercio y servicio), la mayor comparación que se tiene para las herramientas de auditoria tradicionales y especializadas son la capacidad de respuesta de cada una, aunque no son indispensables, una es complemento de la otra para poder dictaminar una empresa con la mayor veracidad y facilidad en la presentación de el dictamen. 
Con estas herramientas se puede evitar la información errónea, evitar los fraudes o espionajes de la información además de que están creadas para alertar de sistemas mal diseñado o el ingreso de usuarios no deseados.

Para diferenciar las causales de riesgo que existe con cada una de las herramientas de auditoria ya sean tradicionales o especializadas es necesario realizar una matriz de riesgo para poder entender cada uno de ellos.

Tabla 2. Riesgos en las herramientas de auditoria.

\begin{tabular}{|c|c|c|c|c|c|c|}
\hline \multicolumn{7}{|c|}{$\begin{array}{c}\text { MATRIZ DE RIESGO } \\
\text { HERRAMEINTAS DE AUDITORÍA EN UN PERTTAJE CONTABLE }\end{array}$} \\
\hline $\begin{array}{l}\text { Tipo de } \\
\text { Herrameinta }\end{array}$ & Valoración & Nivel & Norma & Tipo de Riesgo & Porcentaje & $\begin{array}{l}\text { Clase de } \\
\text { Riesgo }\end{array}$ \\
\hline Entrevista & Media & 2 & NIA 510 & $\begin{array}{l}\text { Se porduce cuando el personal a cargo } \\
\text { de la auditoría omite o pregunta de una } \\
\text { forma no correcta para la obtención de } \\
\text { la información vital para la auditoría }\end{array}$ & $40 \%$ & $\begin{array}{l}\text { Riesgo } \\
\text { Humano }\end{array}$ \\
\hline Cuestionarios & Medio & 2 & NIA 300 & $\begin{array}{l}\text { Se produce cuando el auditor } \\
\text { fundamenta bien las preguntas que } \\
\text { estaran en el cuestionario y con esto no } \\
\text { cumplirá su objetivo trazado }\end{array}$ & $40 \%$ & $\begin{array}{l}\text { Riesgo } \\
\text { Humano }\end{array}$ \\
\hline $\begin{array}{l}\text { Trazas o } \\
\text { Huellas }\end{array}$ & Alto & 3 & NIA 510 & $\begin{array}{l}\text { Sin una entrevista o sin un cuestrionario } \\
\text { bien fundamentado, la traza o huella se } \\
\text { puede decir que srá una herramienta } \\
\text { que fallará debido a que esta se utiliza } \\
\text { para validar y comprobar la ejecución } \\
\text { de su trabajo. }\end{array}$ & $60 \%$ & $\begin{array}{l}\text { Riesgo } \\
\text { Humano }\end{array}$ \\
\hline Muestreo & Bajo & 1 & NAI 510 & $\begin{array}{l}\text { El auditor no se encuentra obligado a } \\
\text { examinartodas las áreas de la empresa, } \\
\text { pero gracias a su experiencia este sabrá } \\
\text { por dónde empezar con el muestreo } \\
\text { para obtener la información adecuada. }\end{array}$ & $20 \%$ & $\begin{array}{l}\text { Riesgo } \\
\text { Humano }\end{array}$ \\
\hline $\begin{array}{l}\text { Cuaderno de } \\
\text { campo }\end{array}$ & Medio & 2 & NAI 510 & $\begin{array}{l}\text { Con esta herramienta el auditor toma } \\
\text { nota de todos los hallazgos } \\
\text { encontrados pero en el momento que } \\
\text { llegue a pasar por alto algun detalle por } \\
\text { mínimo que sea su dictamen final } \\
\text { puede variar }\end{array}$ & $40 \%$ & $\begin{array}{l}\text { Riesgo } \\
\text { Humano }\end{array}$ \\
\hline
\end{tabular}




\begin{tabular}{|c|c|c|c|c|c|c|}
\hline \multicolumn{7}{|c|}{$\begin{array}{c}\text { MATRIZ DE RIESGO } \\
\text { HERRAMEINTAS DE AUDITORÍA EN UN PERITAJE CONTABLE }\end{array}$} \\
\hline $\begin{array}{l}\text { Tipo de } \\
\text { Herrameinta }\end{array}$ & Valoración & Nivel & Norma & Tipo de Riesgo & Porcentaje & $\begin{array}{l}\text { Clase de } \\
\text { Riesgo }\end{array}$ \\
\hline \multicolumn{7}{|c|}{ RIESGOS EN LAS HERRAMIENTAS ESPECIALIZADAS DE AUDITORÍA } \\
\hline $\begin{array}{l}\text { Software de } \\
\text { auditoría }\end{array}$ & Baja & 1 & NIA 230 & $\begin{array}{l}\text { Debido a que son herramientas } \\
\text { mundialmente reconocidas es muy } \\
\text { bajo el porcentaje de riesgo o falla } \\
\text { que puede tener esta herrameinta } \\
\text { sistematizada. }\end{array}$ & $5 \%$ & $\begin{array}{l}\text { Riesgo de } \\
\text { sistema }\end{array}$ \\
\hline Siniestro & Baja & 1 & NIA 240 & $\begin{array}{l}\text { Puede ocurrir cuando se llegase a } \\
\text { presentar fuego, inundación o alguna } \\
\text { clase de desastre natural y con esto se } \\
\text { perdería toda la información }\end{array}$ & $10 \%$ & $\begin{array}{l}\text { Riesgo } \\
\text { Inherente }\end{array}$ \\
\hline
\end{tabular}

En los delitos económicos o financieros representados en el Código Penal, por lo general es necesaria la actuación de peritos contables como auxiliares de la justicia. De esta manera, el profesional designado elabora un informe pericial de su trabajo de análisis e investigación de evidencia y comparece en el juicio oral y público para que pueda ser interrogado y contrainterrogado.

La actuación del profesional de la contaduría pública como perito y auxiliar de la justicia se relaciona con los delitos económicos y financieros, así:

\section{Tabla 3. Delitos económicos y financieros en Colombia}

\begin{tabular}{ll}
\hline $\begin{array}{l}\text { Delitos contra el orden económico social } \\
\text { (Título X CP) }\end{array}$ & $\begin{array}{l}\text { El Acaparamiento, la especulación y otras infracciones, delitos } \\
\text { contra el sistema financiero, urbanización ilegal, contrabando y } \\
\text { lavado de activos (Capítulos I-V). }\end{array}$ \\
$\begin{array}{l}\text { Delitos contra el patrimonio económico } \\
\text { (Título VII CP) }\end{array}$ & $\begin{array}{l}\text { Hurto, extorsión, estafa, fraude mediante cheque, del abuso de } \\
\text { confianza, defraudaciones, usurpación, daño en bien ajeno } \\
\text { (Capítulos I-IX) }\end{array}$ \\
$\begin{array}{l}\text { Delitos contra la administración Pública } \\
\text { (Título XV CP) }\end{array}$ & $\begin{array}{l}\text { Peculado, concusión, cohecho, celebración indebida de } \\
\text { contratos, delitos contra los empleados oficiales (Capítulos I-X) }\end{array}$
\end{tabular}

Fuente- Ramírez, Robayo, y Parra, $(2015$, p.697)

El último elemento que hay que evaluar para señalar diferencias, es el resultado obtenido de cada uno de los procesos de auditoría. Bajo el tono de la auditoría convencional, los resultados están enmarcados en el dictamen sobre los estados financieros, acompañados de una carta de control interno; en la auditoría moderna en las NIA, el resultado es el dictamen, perfil de riesgos de los 
negocios, análisis de brechas de situaciones y escenarios, sugerencias para el mejoramiento de rendimientos y controles, es decir, bajo NIA se otorgan valores agregados a la entidad auditada, teniendo como principal característica el propósito de los resultados por ser preventivos y no correctivos, puesto que el enfoque en el riesgo permite la previsión que disminuye los efectos negativos sobre la organización.

\section{CONCLUSIONES}

$\mathrm{L}$ a auditoría forense es una auditoría especializada en allegar la prueba pericial contable especializada ante los tribunales judiciales, el objetivo general de la investigación fue la valoración de las herramientas de auditoría aplicables al peritaje contable junto con la medición de los riesgos que cada una conlleva.

El objeto de estudio se fundamentó en la forma en que los contadores públicos y los facultados para ejercer la auditoria usan y conocen las herramientas de auditoría aplicables en un peritaje contable.

Como primera conclusión y la más importante se tiene, que la auditoría es una herramienta que se aplica en cualquier momento y a cualquier entidad y que ayuda a la gestión de la empresa a evaluar su sistema, un proceso o subproceso obteniendo resultados que promuevan la reducción de costos.

En el primer objetivo específico que se analizó fue la valoración de las herramientas de auditoría, aplicables al peritaje contable, la investigación dio a conocer el nivel de riesgo que tiene cada herramienta de auditoría cuando el auditor falla sea por cuenta propia o por presión del cliente, esto se da, debido a el tipo de riesgo que maneja cada auditor cuando hace un uso indebido de las herramientas de auditoría o un desconocimiento de las mismas.

En cuanto al segundo objetivo específico se realizó la comparación entre las herramientas de auditorías tradicionales y especializadas dando a conocer que tan importantes y complementarias son las herramientas ya que cumplen un objetivo específico en ayudar a la realización de un dictamen más verídico.

La investigación de alguna forma pretende dar a conocer la importancia de la informática forense, debido a que esta ciencia integra otros conceptos como: auditoría, aspectos legales que se enmarcan en el perfil profesional integral. 


\section{REFERENCIAS}

Actualices, (2017). Cuestionarios de auditoría, una herramienta importante para el control interno. (2017, 21 junio). Recuperado de https://actualicese.com/2017/06/21/cuestionariosde-auditoria-una-herramienta-importante-para-elcontrol-interno/

Alatrista, M. (2015). Técnicas y Procedimientos de Auditoría. Lo que todo auditor debe conocer. Recuperado de https://www.auditool.org/blog/auditoriaexterna/2158-tecnicas-y-procedimientos-deauditoria-lo-que-todo-auditor-debe-conocer

Contraloría de Bogotá. (s.f.). Procedimiento para la elaboración del memorando de planeación y programas de auditoria

Contraloría de Bogotá. (2012). Procedimiento para la elaboración del memorando de planeación y programas de auditoria

Cuellar, G. (2017). Contratos de aseguramiento. Recuperado de http://fccea.unicauca.edu.co/old/aseguramiento.ht $\mathrm{m}$

Duque, F. y Tamayo, A. (2012). Auditoría con Informática a Sistemas Contables. Recuperado de https://www.researchgate.net/profile/Yeiniel_Alfons o2/publication/277274628_Auditoria_con_Informati ca_a_Sistemas_Contables/links/571b126708ae4083 67bc9286/Auditoria-con-Informatica-a-SistemasContables.pdf

Frett, N. (2016). Técnicas para el muestreo en auditoría. Recuperado de https://www.auditool.org/blog/auditoriainterna/4267-tecnicas-para-el-muestreo-enauditoria

Flórez, M.; Gallego, L., y Hernández, L. (2015). Tableros de control como herramienta especializada.
Revistas Javeriana, 1, 665. Recuperado de http://revistas.javeriana.edu.co/index.php/cuacont/ article/view/17387

Ley 43 de 1990. (1990). Reglamento de la profesión de Contador Público. Recuperado de https://nif.com.co/ley-43-1990/

Martínez, Y. A., Alfonso, B. B., y Marichal, L. L. (2012). Auditoría con informática a sistemas contables. Revista Arquitectura e Ingeniería, 6(2), 414.Ministerio de comercio, industria y turismo. (s.f.). DECRETO 2170 [varios]. Recuperado de https://nif.com.co/decreto-2170-2017/

Ramírez, M.; Robayo, N., y Parra, L. (2015). La prueba pericial contable especializada en los delitos económicos y financieros: análisis del caso DMG. Recuperado 13 diciembre, 2017, de http://www.scielo.org.co/pdf/cuco/v16nspe42/v16n spe42a05.pdf

Tello, M. (2012). Manual de auditoria de la comunicación interna. Recuperado de http://www.maxtello.com/clase4tcomorgentrev.pdf

Martínez, Y. A., Alfonso, B. B., y Marichal, L. L. (2012). Auditoría con informática a sistemas contables. Revista Arquitectura e Ingeniería, 6(2), 414.Ministerio de comercio, industria y turismo. (s.f.). DECRETO 2170 [varios]. Recuperado de https://nif.com.co/decreto-2170-2017/

Ramírez, M.; Robayo, N., y Parra, L. (2015). La prueba pericial contable especializada en los delitos económicos y financieros: análisis del caso DMG. Recuperado 13 diciembre, 2017, de http://www.scielo.org.co/pdf/cuco/v16nspe42/v16n spe42a05.pdf

Tello, M. (2012). Manual de auditoria de la comunicación interna. Recuperado de http://www.maxtello.com/clase4tcomorgentrev.pdf 